\title{
O ESTABELECIMENTO DE PADRÕES DE REFERÊNCIA ALTIMÉTRICA UTILIZANDO O NIVELAMENTO GEOMÉTRICO PARA A DEFINIÇÃO DE ALVOS ALTOS E INACESSÍVEIS
}

The establishment of standards of measurement using geometric leveling for definition of high and inaccessible points

\author{
ANDRÉA DE SEIXAS ${ }^{1}$ \\ LUCIENE FERREIRA GAMA ${ }^{2}$ \\ JOÃO NAVES DE MORAES ${ }^{3}$ \\ ANGELA MARIA BARBOSA DE SOUZA ${ }^{1}$ \\ ${ }^{1}$ Universidade Federal de Pernambuco - UFPE \\ Centro de Tecnologia e Geociências - CTG \\ Departamento de Engenharia Cartográfica, Recife, PE \\ Programa de Pós-Graduação em Ciências Geodésicas e Tecnologias da \\ Geoinformação \\ ${ }^{2}$ Instituto Federal de Educação, Ciência e Tecnologia da Paraíba - IFPB \\ Unidade Acadêmica de Design, Infraestrutura e Meio Ambiente \\ Curso Superior de Tecnologia em Geoprocessamento \\ ${ }^{3}$ Universidade de São Paulo - USP \\ Escola Politécnica da Universidade de São Paulos - EPUSP-PTR \\ Laboratório de Topografia e Geodésia \\ CEP: 05424-970, São Paulo - SP \\ aseixas@ufpe.br; angelabarbosa2000@yahoo.com.br; \\ Luciene.gama@ifpb.edu.br; j_moraes@gmail.com
}

\begin{abstract}
RESUMO
As estruturas geodésicas altimétricas são fundamentais para os projetos de Engenharia. Neste contexto, pode-se utilizar a tecnologia do nível digital para a determinação de pontos de referência altimétrica através do método do nivelamento geométrico. Outra solução é a utilização da tecnologia da estação total para a determinação de altitudes em pontos situados em locais altos e inacessíveis nas
\end{abstract}


estruturas arquitetônicas, como por exemplo, alvos em estrutura de edificação situados em torres de igrejas e para a realização de uma estrutura geodésica através do método de nivelamento trigonométrico. As estruturas geodésicas altimétricas, implantadas e determinadas pelo método do nivelamento geométrico, materializam, neste trabalho, pontos para o estabelecimento de padrões de referência altimétrica e para a determinação altimétrica de alvos altos e inacessíveis. As estruturas geodésicas foram implantadas e determinadas, no Sítio Histórico de Olinda, empregando-se nível digital de alta precisão e mira de ínvar com código de barras. Este trabalho tem como objetivo definir padrões de referência altimétrica utilizando o método de nivelamento geométrico, resultando na implantação e análise da qualidade de estruturas geodésicas altimétricas.

Palavras-chave: Estruturas Geodésicas Altimétricas; Levantamentos Geodésicos; Nivelamento Geométrico de Alta Precisão.

\begin{abstract}
The altimetric geodesic structures are noteworthy for engineering projects. In this context, the technology of digital level can be used for the definition, by means of the geometric leveling method, of altimetric reference points. Another solution is the use of total station technology for determining inaccessible high points in the architectural structures, for example, structure targets of a building placed in towers of churches and builders of a geodesic structure through the trigonometric leveling method. The altimetric geodesic structures, established and determined by the geometric leveling method, materialize, in this work, of points for the establishment of standards of altimetric reference and determination of high and inaccessible points. The geodesic structures were established and determined, in Olinda's Historical Site, by using digital level of high accuracy and invar rod with barcode. This work aims at defining altimetric reference standards using the geometric leveling method, resulting in the implementation and analysis of the quality of altimetric geodesic structures.
\end{abstract}

Keywords: Altimetric Geodesic Structures; Geodesic Surveying; Geometric Leveling of High Precision.

\title{
1. INTRODUÇÃO
}

As estruturas geodésicas altimétricas são fundamentais para os projetos de Engenharia, tais como: locação e levantamento de estruturas de edificações prediais, levantamentos cadastrais, traçados de rodovias e ferrovias, posicionamento e controle de máquinas, assim como, monitoramento de estruturas geodésicas, pois definem uma referência altimétrica. Estas permitem em conjunto com as referências planimétricas a reconstrução 3D de objetos, fundamental para o estudo geoespacial das cidades e suas edificações.

Uma dificuldade frequente na reconstrução tridimensional e nos levantamentos topográficos/geodésicos é a escassez de pontos de referência planialtimétricos nas 
proximidades dos imóveis urbanos e rurais brasileiros. Uma solução para essa dificuldade é a utilização do sistema GNSS (Global Navigation Satellite System Sistema Global de Navegação por Satélite) integrado a medições terrestres planialtimétricas para definição de um conjunto de pontos de referência, que definem geometricamente os elementos levantados sobre a superfície do terreno e do objeto (natural ou artificial), em um determinado sistema de referência.

A determinação de pontos situados em locais altos e inacessíveis nas estruturas arquitetônicas, como por exemplo, alvos da estrutura de uma edificação situados em torres de igrejas, é importante, pois apesar de serem inacessíveis são visíveis de outras localidades e mais difíceis de serem obstruídos por ações antrópicas.

No contexto deste trabalho será abordada a definição de estruturas geodésicas implantadas por meio do método do nivelamento geométrico de alta precisão conforme norma para execução de Levantamentos Topográficos (NBR 13.133/1994). Estas estruturas altimétricas materializam um conjunto de pontos para o estabelecimento de padrões de referência altimétrica que auxiliam na determinação de altitudes de alvos altos e inacessíveis. Os experimentos foram realizados na área do Sítio Histórico de Olinda, denominada Área Teste. Nesta foi contemplada a implantação e definição de um conjunto de estruturas geodésicas altimétricas com o emprego de nível digital de alta precisão $( \pm 0,3 \mathrm{~mm} / \mathrm{km})$. Estes pontos de referência altimétrica permitem realizar Levantamentos Topográficos/Geodésicos e Reconstrução 3D de objetos das respectivas áreas e seus entornos. Neste sentido, o Sítio Histórico de Olinda foi contemplado com a implantação de novas estruturas geodésicas altimétricas com referência ao Datum Vertical Brasileiro através da RN394D do IBGE.

\section{ESTRUTURAS GEODÉSICAS ALTIMÉTRICAS IMPLANTADAS COM NÍVEL DIGITAL}

A seguir serão abordadas a definição e implantação de estruturas geodésicas de alcance local com o método do nivelamento geométrico. A qualidade das altitudes será determinada através da propagação das covariâncias após o ajustamento realizado pelo Método dos Mínimos Quadrados. Este método aceita como melhor estimativa de uma grandeza $\mathrm{X}$ o valor que torna mínima a soma dos quadrados dos resíduos (MIKHAIL, 1981).

\subsection{Definição e Implantação}

Para a implantação das Referências de Nível (RRNN) foi realizado anteriormente um reconhecimento da área, a fim de escolher o melhor local de acordo com a localização dos vértices geodésicos implantados com receptores GNSS e Estação Total e de acordo com as localidades das Igrejas, Figura 1, cujas torres seriam levantadas (SOUZA, 2012). A Figura 2 apresenta em vista aérea um esboço da configuração das RRNN. 
Figura 1 - Alvos nas torres das Igrejas: Nossa Senhora da Misericórdia, Igreja da Sé e Igreja de Nossa Senhora do Carmo. Fonte: SEIXAS et al., (2013). Fotos: Outubro 2011.

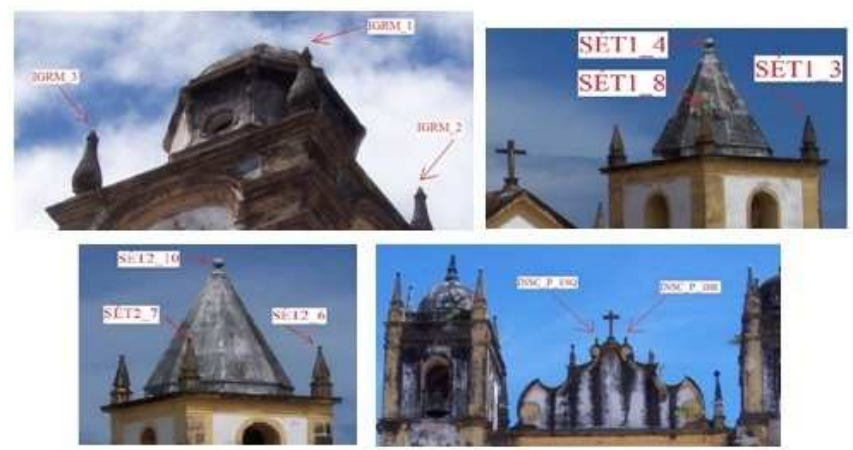

Figura 2 - Vista aérea da configuração dos pontos de referência planialtimétrica. Fonte: Adaptada Google Earth (2009).

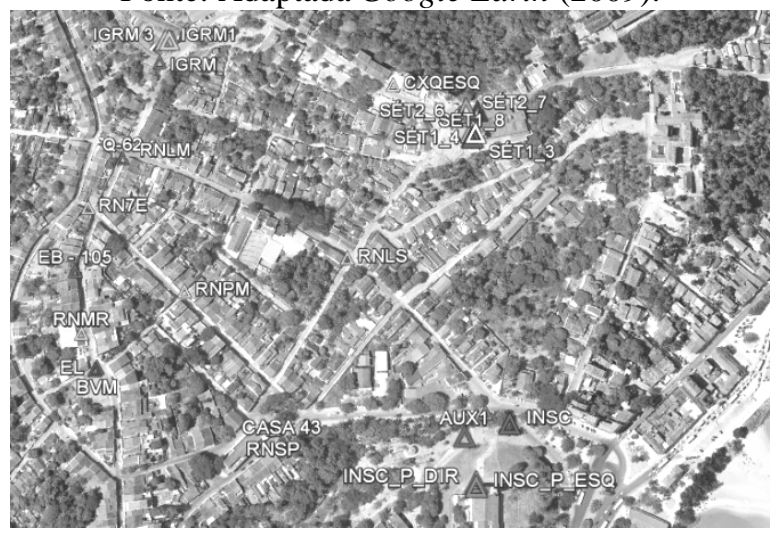

Os pontos de referência para a execução do nivelamento geométrico é composto por seis RRNN: Referência de Nível do IBGE (RN394D), Referência de Nível Igreja Nossa Senhora do Carmo (RNSC), Referência de Nível Ladeira da Sé $(R N L S)$, Referência de Nível Sétima Estação da Via Sacra $(R N 7 E)$, Referência de Nível Rua Prudente de Moraes (RNPM), Referência de Nível Mercado da Ribeira $(R N M R)$. As novas RRNN implantadas foram materializadas com pinos de superfícies aboladas esféricas, Figura 3, fixadas entre os meios fios e envolvidas com adesivo de alta resistência. Além disso, foi aproveitada uma estrutura de metal fixada na esquina entre as ruas Ladeira da Misericórdia e Bonfim para definir a sétima Referência de Nível: Referência de Nível denominada Ladeira da Misericórdia (RNLM), Figura 4. 
Figura 3-Modelo do Pino das novas Referências de Nível.

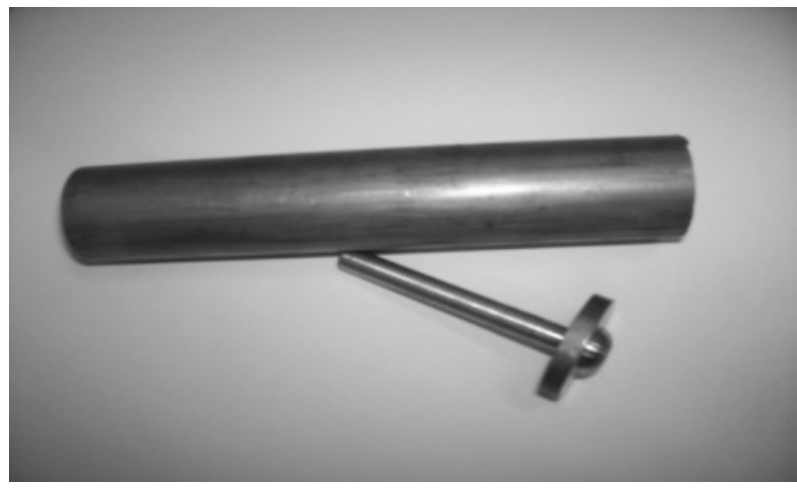

Figura 4 - Estrutura Metálica da RN Ladeira da Misericórdia.

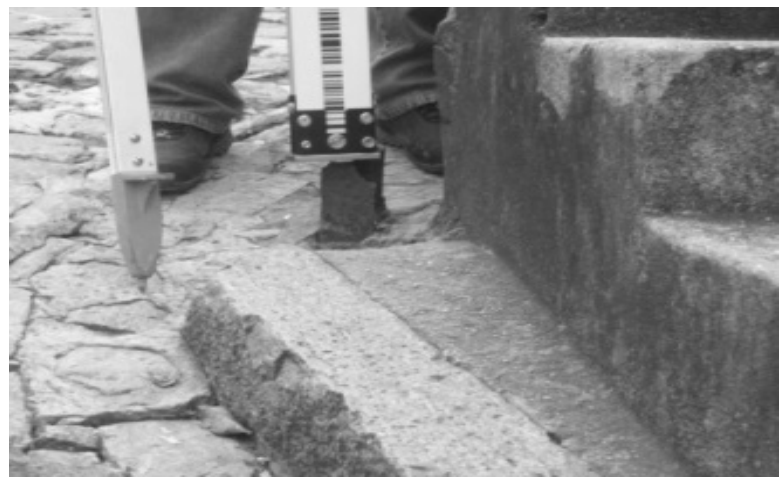

Neste trabalho serão contempladas estruturas geodésicas de alcance local. Segundo Torge (2003), estas são implantadas principalmente para projetos de engenharia, investigações geofísicas e para a determinação de procedimentos geodinâmicos espacialmente limitados.

Exemplos de estruturas geodésicas de alcance local empregadas na engenharia, como, monitoramento da subsidência do solo; racionalização construtiva no posicionamento preciso de edificações prediais; monitoramento de encosta, levantamentos cadastrais urbanos e definição de geóide local, respectivamente, são abordados em (SANTOS, 2005; BOTELHO, 2003; RHADAMEK, 2008; GAMA, 2008 e GUIMARÃES, 2013).

\subsection{Método de Medição: Nivelamento Geométrico}

O nível é um instrumento geodésico/topográfico de medição que tem a finalidade de realizar em campo um plano horizontal. Ele possui algumas características geométricas básicas que devem ser verificadas e testadas. Até mesmo 
durante seu manuseio, a configuração geométrica de seus eixos, poderá se modificar, tendo assim que se submeter sempre a verificações para poder garantir os resultados das observações de campo e minimizar os inevitáveis erros sistemáticos durante seu manuseio. Por isto, as medições são ordenadas de forma a evitar que pequenos erros influenciem os resultados das observações (DEUMILICH; STAIGER, 2002).

A altimetria reproduz a verdadeira forma do terreno e métodos de nivelamento são empregados para a determinação das diferenças de nível (JORDAN, 1944).

O Nivelamento Geométrico é o método que determina a diferença de nível entre pontos do terreno através de visadas horizontais, em miras posicionadas na vertical sobre os pontos, determinadas com um nível (NBR 13.133/1994).

Será denominada altitude nivelada àquela resultante da operação de nivelamento geométrico (FREITAS; BLITZKOW, 1999). Este consiste em realizar leituras em miras graduadas, ré e vante, utilizando um nível de luneta, Figura 5. O nível deve estar nivelado, portanto, estar com seu eixo óptico tangente à superfície equipotencial que passa pelo mesmo (DREWES et al., 1998).

Para determinar a altitude do ponto B, Figura 5, acima da superfície de referência definida pela Referência de Nível A, a diferença de nível $\Delta h_{A B}$ é determinada a partir dos trechos de nivelamento simples. Para isso a distância $\mathrm{AB}$ é subdividida por lances separados por pontos de mudanças $W_{1}, \ldots, W_{2}$ de no máximo $100 \mathrm{~m}$, sendo as diferenças de nível individuais $h_{1}, h_{2}, \ldots, h_{n}$ observadas e adicionadas umas às outras (KAHMEN, 2005).

$$
\Delta h_{A B}=h_{1}+h_{2}+\cdots=\sum h=\sum R e ́-\sum \text { Vante }
$$

Figura 5 - Lances de nivelamento entre os ponto A e B. Fonte: KAHMEN (2005).

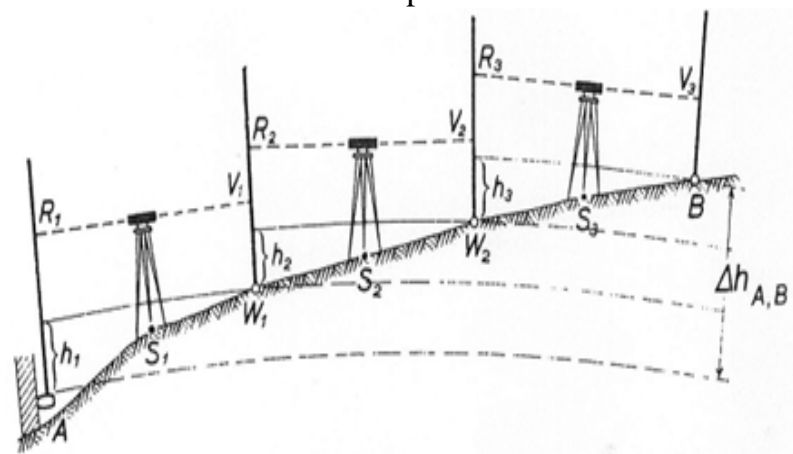

Os procedimentos de verificação de níveis de luneta, quanto ao paralelismo e horizontalidade do eixo óptico da luneta e do eixo do nível de bolha estão descritos em (DEUMILICH; STAIGER, 2002; KAHMEN, 2005). 
2.2.1 Controle de Qualidade trabalho:

O controle de qualidade do nivelamento geométrico foi avaliado neste

- A partir da verificação do nível de luneta empregado;

- Em loco em função das observações realizadas em campo e seus respectivos desvios;

- A partir do cálculo do erro de fechamento nos circuitos de nivelamento geométrico e respectivos perímetros realizados;

- A partir do emprego de visadas equidistantes.

\subsubsection{Método dos Mínimos Quadrados- Modelo dos Correlatos}

Ao realizar um nivelamento geométrico ao longo de um circuito fechado, a soma dos desníveis deveria ser teoricamente nula. Os erros de medição proporcionam erros de fechamento, que servem como indicador preliminar da precisão das medidas e devem ser distribuídos no ajustamento (GEMAEL, 1994; NIEMEIER, 2002).

O erro de fechamento no nivelamento geométrico de alta precisão é de $\leq 3 \mathrm{~mm}$ (L) $1 \frac{1}{2}$, sendo L o perímetro do circuito durante a medição em km (IBGE, 1983). Para valores de fechamento maiores que este, recomenda-se um novo levantamento.

$\mathrm{O}$ transporte de altitudes é feito através das observações ajustadas. O Modelo dos Correlatos, também denominado Modelo das Equações de Condição, é o modelo utilizado no ajustamento quando se tratam de observações, sujeitas a equações de condição. $\mathrm{O}$ modelo matemático que caracteriza as observações condicionadas é descrito por:

$$
F\left(L_{a}\right)=0 \text {, }
$$

que compreende o conjunto de equações de condição a serem satisfeitas pelas observações ajustadas. $\mathrm{O}$ modelo dos correlatos não trata de parâmetros, abrange apenas valores observados ajustados no qual $F$ representa $r$ funções e o vetor $L_{a}$ tem dimensão $n \times 1$ (GEMAEL, 1994).

Envolvendo os valores observados no modelo, Equação 2, obtêm-se as equações de condição transformadas, acrescidas dos resíduos $V_{i}$, Equação 3.

$$
L_{a}=L_{b}+V
$$

Pode-se assim, representar o modelo matemático por:

$$
F\left(L_{b}+V\right)=0
$$

Empregando-se a linearização da série de TAYLOR, na forma matricial, obtémse a Equação 5.

$$
F\left(L_{a}\right)=F\left(L_{a}+V\right) \approx F\left(L_{b}\right)+\left.\frac{\partial F}{\partial L_{a}}\right|_{b} .\left(L_{a}-L_{b}\right)=0
$$

A função $F\left(L_{b}\right)$, dos valores observados, representa um erro de fechamento e é denominada por: 


$$
W=F\left(L_{b}\right)
$$

Denominando-se por B a matriz das derivadas parciais, ou seja,

$$
B=\left.\frac{\partial F}{\partial L_{a}}\right|_{L_{b}}
$$

que corresponde ao modelo linearizado dos correlatos, envolvendo $r$ equações de condição transformadas com $n$ incógnitas.Tendo-se as dimensões descritas por:

$$
{ }_{r} B_{n^{n}} V_{1}+{ }_{r} W_{u}={ }_{r} 0_{1}
$$

\subsubsection{Análise da qualidade do ajustamento}

A Análise da Qualidade do Ajustamento é baseada na comparação entre $\widehat{\sigma}_{0}^{2}$ (variância da unidade de peso a posteriori) e $\sigma_{0}{ }^{2}$ (variância da unidade de peso a priori). Para esta análise é utilizado o teste Qui-Quadrado $\left(\chi^{2}\right)$ conhecido também como Teste Global do Modelo (TGM) ou Teste n-dimensional (MIKHAIL; GRACIE, 1981; FRASER, 2003).

O teste leva a formação de duas hipóteses:

$$
\left\{\begin{array}{l}
H_{0}: E\left\{\hat{\sigma}_{0}^{2}\right\}=\sigma_{0}{ }^{2} \\
H_{1}: E\left\{\hat{\sigma}_{0}^{2}\right\}>\sigma_{0}{ }^{2}
\end{array}\right.
$$

Para a validação de uma das hipóteses, compara-se o valor calculado dado por:

$$
T=\chi_{c}^{2}=\frac{\hat{\sigma}_{0}^{2}}{\sigma_{0}{ }^{2}} S
$$

Onde $T$ tem distribuição $\chi^{2}$, com graus de liberdade igual a $n$ - $u$, com os valores teóricos da distribuição $\chi_{(S, 1-\alpha)}^{2}$. A hipótese nula $H_{0}$ não é rejeitada, ao nível de significância $\alpha$, no teste estatístico, se: $T<\chi_{(S ; 1-\alpha)}^{2}$. Caso contrário, $T>\chi_{(S ; 1-\alpha)}^{2}$, significa que há problemas no ajustamento, ou seja, a hipótese alternativa é rejeitada ao nível de significância de $\alpha$. Desta forma, o ajustamento não é aceito, pois existem erros envolvidos.

Segundo Gemael (1994), as possíveis causas do ajustamento não ser aceito podem ser:

- O modelo funcional não representa a realidade física;

- A Matriz Variância-Covariância das observações não está apropriadamente determinada (reponderar as observações);

- Erros grosseiros ou sistemáticos presentes no ajustamento.

Desta forma, torna-se necessário realizar um teste estatístico para identificar as possíveis observações com erros grosseiros ou sistemáticos. 
O teste é baseado no resíduo e na sua Matriz Variância-Covariância. O resíduo que contradiz uma propriedade estatística $E\{v\}=0$ é designado outlier.

Alguns dos métodos para testar a presença de um outlier na observação são citados em (MONICO, 2008; MONICO, SILVA, 2003; GEMAEL, 1994; MENDONÇA et al., 2010) são:

- $\quad$ Método de Baarda (BAARDA, 1968) - Data Snooping (Teste w);

- Método de POPE (POPE, 1976) - Teste Tau;

- Danish Method (KRARUP e KUBIK, 1982).

Neste trabalho, utilizou-se o Método de Baarda que trata da convenção de que somente um outlier está presente em cada teste. Assim, ao testar a presença de um outlier na observação (i), o teste de hipóteses assume a seguinte forma (MONICO; SILVA, 2003):

$$
H_{0}=E\{L\}=A X \operatorname{contra} H_{a_{i}}=E\{L\}=\left(\begin{array}{ll}
A & C_{L_{i}}
\end{array}\right)\left(\begin{array}{l}
X \\
\nabla_{i}
\end{array}\right) ; \nabla_{i} \neq 0
$$

Onde

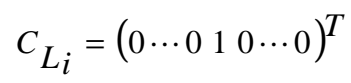

Para aplicar o Método de Baarda, devem-se conhecer os resíduos, bem como a sua Matriz Variância-Covariância, pois neste método, calculam-se os resíduos normalizados da seguinte forma:

$$
W=\frac{C^{T} \cdot P \cdot V}{\sqrt{C^{T} \cdot P \cdot \sum V \cdot P . C}}
$$

Onde C é um vetor n-dimensional, contendo elementos unitários para as observações a serem testadas e zero para as demais posições.

$$
C=\left(\begin{array}{c}
0 \\
0 \\
\vdots \\
1 \\
0
\end{array}\right)_{n \times 1}
$$

Quando a Matriz Variância-Covariância dos resíduos é diagonal, pode-se calcular o valor de $w$ para cada observação.

$$
w_{i}=\frac{v_{i}}{\sigma_{i}}, \operatorname{com} 1<\mathrm{i}<\mathrm{n} ;
$$

na qual:

$w_{i}$ é o resíduo normalizado; $v_{i}$ o resíduo da i-ésima observação; $\sigma_{i}$ o desvio padrão do resíduo da i-ésima observação. 
Considerando que a Matriz Variância-Covariância dos resíduos deve ser calculada usando o fator de variância a priori, têm-se:

$$
\sum V=\sigma_{0}^{2} \cdot P^{-1} \cdot B^{T} \cdot M^{-1} \cdot B \cdot P^{-1}
$$

Para a não rejeição de uma hipótese deve-se comparar o valor teórico $\left(-N_{\frac{\alpha_{o}}{2}} ; N_{\frac{\alpha_{o}}{2}}\right)$, onde $N$ representa a Distribuição Normal, com o valor calculado $w$.

A estatística $w_{i}$ tem distribuição $F$ de Snedecor $\sqrt{F}$ com graus de liberdade $1 \mathrm{e}$ $\infty, \sqrt{F_{1, \infty}}$ ou $w_{i} \sim N_{\frac{\alpha}{2}(0,1)}$ onde $\alpha$ indica o nível de significância.

Hipóteses:

$$
\left\{\begin{array}{l}
H_{0}: v_{i}=0 \\
H_{1}: v_{i} \neq 0
\end{array}\right.
$$

A hipótese básica $H_{0}$ não é rejeitada a um determinado nível de significância $\alpha_{o}=\frac{\alpha}{n}$, se: $-N_{\frac{\alpha o}{2}}<w_{i}<N_{\frac{\alpha o}{2}} o u-\sqrt{\chi_{\alpha o, 1}^{2}}<w_{i}<\sqrt{\chi_{\alpha o, 1}^{2}}$

Caso contrário, a observação $i$ contém erro grosseiro. Detectado mais de uma observação com erro será eliminada a que representar a maior estatística $\left|w_{i}\right|$, pois o teste é unidimensional. O teste deve ser repetido até que todas as observações com erro sejam eliminadas. Portanto, o intervalo será:

$$
-N_{\frac{\alpha o}{2}}<w_{i}<N_{\frac{\alpha o}{2}}
$$

Reiniciando-se um novo ajustamento.

\section{EXPERIMENTOS E ANÁLISES DAS ESTRUTURAS GEODÉSICAS ALTIMÉTRICAS}

\subsection{Materiais}

Na obtenção dos dados de campo foram utilizados o nível digital da marca Leica DNA - 03 e mira de ínvar com código de barras de $2 \mathrm{~m}$. Segundo Leica (2002), este nível digital possibilita a medição eletrônica da altura, bastando apenas um nivelamento aproximado através de um nível de bolha. O compensador de alta precisão realiza automaticamente o ajustamento fino do feixe de LASER. Acionando-se uma tecla a medição é efetuada. O código de barras da mira é capturado no instrumento como sinal de referência. No processo de medição, o decodificador de linhas armazena e interpreta como um sinal de medição a seção da mira no campo de visão, o sinal da medição é analisado com o sinal de referência. Esta análise possibilita a determinação do valor da altura e da distância horizontal. A mira deve estar na posição vertical durante a medição. É possível realizar medições no escuro através de iluminação artificial sobre a mesma. A medição de 
altura com mira de ínvar tem uma precisão nominal de $\pm 0,3 \mathrm{~mm} / \mathrm{km}$ e a medição de distância tem um desvio de $5 \mathrm{~mm} / 10 \mathrm{~m}$. O tempo de medição é de aproximadamente 3 segundos (LEICA, 2002).

Conforme a NBR 13.133/94 (ABNT,1994), que apresenta quatro categorias de classes de níveis, a classificação do nível digital DNA - 03 utilizado, segundo o desvio padrão de $1 \mathrm{~km}$ de duplo nivelamento é de precisão muito alta com valor $\leq \pm 1 \mathrm{~mm} / \mathrm{km}$.

\section{3,2 Área Teste - Sítio Histórico de Olinda}

Com localização excêntrica ao Campus Recife da UFPE, a Área Teste está localizada na Cidade de Olinda, Região Metropolitana do Recife. A área levantada integra o Sítio Histórico de Olinda, constituída por edificações históricas de uso residencial. A Figura 2 apresenta a configuração dos vértices terrestres e alvos altos de triangulação localizados nas Torres das Igrejas: Nossa Senhora da Misericórdia, Igreja da Sé e Nossa Senhora do Carmo.

\subsection{Medição, Processamento e Análise dos Resultados dos Pontos de Referência com Nível Digital}

Na Área Teste estão implantadas sete RRNN, como descrito anteriormente, às quais definem os pontos observados por meio do nivelamento geométrico de alta precisão (cf. item 2.2).

Os experimentos foram realizados no entorno de várias quadras urbanas totalmente edificadas. Essa área situa-se no Centro da Cidade Alta de Olinda, à qual contempla apenas uma Referência de Nível conservada próxima à esquerda da porta principal da Igreja de São Pedro (IBGE, 2011).

De acordo com o banco de dados do IBGE, no Município de Olinda existem 4 referências de nível. Destas, apenas a RN394D com marco em situação adequada.

O sistema de referência altimétrico das RRNN implantadas está fixado com base na altitude da $R N 394 D$ do IBGE, que corresponde a 15,9082m de altitude, com data de cálculo em 15/06/2011. A Referência de Nível $R N 394 D$ pertence a um ramal, isto é, uma linha de nivelamento geométrico que não forma circuito, cujos desníveis, portanto, não foram ajustados (IBGE, 2011).

Ressalta-se que, para validar as altitudes determinadas neste trabalho com respeito ao datum vertical brasileiro é necessário realizar novas linhas de nivelamento geométrico a partir de RRNN, cujas precisões sejam conhecidas.

Em estudo realizado por Matos et al., (2012), que trata da validação do MAPGEO 2010, foi constatado que o não conhecimento das precisões das RRNN definidas por nivelamento geométrico, em algumas regiões brasileiras, comprometem a validação do modelo de alturas geoidais.

A seguir são descritas as campanhas de medição realizadas em três dias: 11, 13 e 14/07/2011. A Figura 6 apresenta o circuito do nivelamento geométrico realizado com o nível digital DNA - 03. 
Figura 6 - Circuito de nivelamento geométrico.

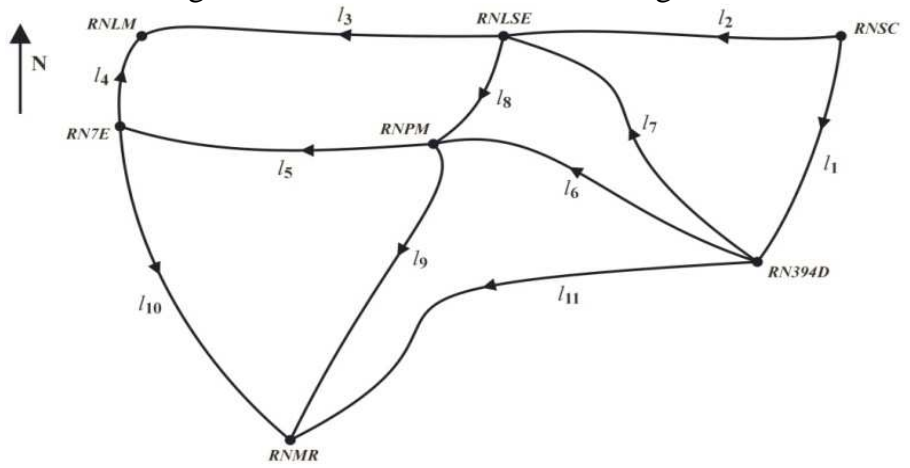

\subsubsection{Medições}

O levantamento foi realizado durante três dias (11,13 e 14/07/2011). As medições com a mira de ínvar de $2 \mathrm{~m}$ foram realizadas continuamente a partir das estações do caminhamento com visadas a ré e a vante. As medições com o equipamento foram realizadas da seguinte maneira: o instrumento estava programado para realizar quatro medições sucessivas, ao término no display eram apresentadas as médias e o desvio padrão da medição da altura e a distância horizontal do equipamento para a mira de ínvar. Durante as medições, a função "EC" estava ativada para a correção da influência da curvatura terrestre. Isto significa que as alturas das miras, medidas eletronicamente, são automaticamente corrigidas em relação à curvatura da Terra.

Com a conclusão do terceiro dia de levantamento foi possível a partir das linhas de nivelamento definidas, fechar vários circuitos de nivelamento, o que permitiu calcular a altitude de uma mesma RN por diversos caminhos de medição como mostrado na Figura 6 e na Tabela 1.

A Tabela 1 apresenta o erro de fechamento para cada circuito independente formado. Foram ao todo oito circuitos calculados, conforme apresentado anteriormente.

Tabela 1- Erro de fechamento e perímetro dos circuitos nivelados.

\begin{tabular}{c|l|c|c|c}
\hline Circuito & \multicolumn{1}{|c|}{ Linhas } & $\begin{array}{c}\text { Perímetro } \\
(\mathrm{km})\end{array}$ & $\begin{array}{c}\text { Erro de } \\
\text { Fechamento(m) }\end{array}$ & Data da Leitura \\
\hline $\mathrm{I}$ & $l_{1}, l_{2}$ e $l_{7}$ & 0,711275 & $-0,00022$ & 11 e $13 / 07 / 2011$ \\
\hline $\mathrm{II}$ & $l_{7}, l_{8} e l_{6}$ & 0,740770 & $-0,00084$ & $11,13 \mathrm{e} 14 / 07 / 2011$ \\
\hline $\mathrm{III}$ & $l_{5}, l_{4}, l_{5}$ e $l_{8}$ & 0,811275 & $-0,00097$ & $13 \mathrm{e} 14 / 07 / 2011$ \\
\hline $\mathrm{IV}$ & $l_{5}, l_{9} e l_{10}$ & 0,49678 & 0,00008 & $13 \mathrm{e} 14 / 07 / 2011$ \\
\hline $\mathrm{V}$ & $l_{6}, l_{9} e l_{11}$ & 0,722760 & $-0,00368$ & $11,13 \mathrm{e} 14 / 07 / 2011$ \\
\hline
\end{tabular}




\subsubsection{Processamento}

Através da distribuição dos erros ocasionados no nivelamento geométrico, foram determinadas as altitudes das RRNN (Referências de Nível).

A Tabela 2 apresenta informações sobre as linhas de nivelamento geométrico realizadas, conforme descrito anteriormente, durante a primeira campanha de medição.

Tabela 2 - Diferenças de nível entre as RRNN e respectivas linhas de nivelamento.

\begin{tabular}{c|c|c|c|c|c}
\hline Saída & Chegada & $\Delta \mathrm{H}(\mathrm{m})$ & Linha & $\begin{array}{c}\mathrm{N}^{\circ} \\
\text { Lances }\end{array}$ & $\begin{array}{c}\text { Comprimento } \\
\text { da linha }(\mathrm{km})\end{array}$ \\
\hline$R N S C$ & $R N 394 D$ & 7,18567 & $l_{1}$ & 9 & 0,260745 \\
\hline$R N S C$ & $R N L S E$ & 9,81698 & $l_{2}$ & 10 & 0,217665 \\
\hline$R N L S E$ & $R N L M$ & 9,03447 & $l_{3}$ & 10 & 0,269565 \\
\hline$R N 7 E$ & $R N L M$ & 7,66104 & $l_{4}$ & 6 & 0,096275 \\
\hline$R N P M$ & $R N 7 E$ & 0,85134 & $l_{5}$ & 5 & 0,153575 \\
\hline$R N 394 D$ & $R N P M$ & 3,15138 & $l_{6}$ & 10 & 0,216045 \\
\hline$R N 394 D$ & $R N L S E$ & 2,63109 & $l_{7}$ & 11 & 0,232865 \\
\hline$R N L S E$ & $R N P M$ & 0,52112 & $l_{8}$ & 12 & 0,291860 \\
\hline$R N P M$ & $R N M R$ & 12,84909 & $l_{9}$ & 9 & 0,156230 \\
\hline$R N 7 E$ & $R N M R$ & 11,99767 & $l_{10}$ & 10 & 0,186975 \\
\hline$R N 394 D$ & $R N M R$ & 15,99680 & $l_{11}$ & 16 & 0,350485 \\
\hline
\end{tabular}

Para o ajustamento da rede de nivelamento utilizou-se como observações a diferença de nível entre as RRNN apresentadas na Tabela 2.

As coordenadas altimétricas foram ajustadas utilizando-se o Método dos Mínimos Quadrados (MMQ) com emprego das equações de condição, ao nível de $99,5 \%$ de confiança. Seguindo-se com a análise da qualidade do ajustamento com o Teste Global do Modelo (TGM).

Foram definidas as matrizes e vetores envolvidos e obedecida à sequência de cálculos utilizada neste modelo (cf. item 2.2.2), finalizando-se com a estimativa das incógnitas e seus graus de confiabilidade, observações ajustadas (ou desníveis ajustados) e altitudes ajustadas. São apresentados ainda o vetor dos resíduos e respectivos graus de confiabilidade além dos resíduos normalizados (cf. item 2.2.3).

Foram realizadas onze linhas de nivelamento geométrico, Figura 6, representando onze desníveis medidos, considerados neste modelo de ajustamento como onze observações $\left(l_{b}\right)$. As linhas de nivelamento geométrico envolveram sete RRNN, sendo a altitude da $R N 394 D$ considerada fixa e as demais consideradas como incógnitas. Resulta assim, que as equações de condição, denominadas de $S=n-u$, sendo $n$ o número de observações e $u$ o número de incógnitas, igual a cinco. 
Para escrever as equações de condição com base na Equação 2, é necessário que as equações sejam independentes entre si. Para efeito de simplificação foram feitas as seguintes considerações: $R N 394 D\left(H_{1}\right), R N S C\left(H_{2}\right), R N L S E\left(H_{3}\right), R N L M$ $\left(H_{4}\right), R N 7 E\left(H_{5}\right), R N P M\left(H_{6}\right), R N M R\left(H_{7}\right)$.

São expressas as equações, utilizando-se os valores das diferenças de nível entre as RRNN, apresentadas na Tabela 2.

Em seguida escrevem-se as equações de condição transformadas adicionadas aos resíduos $v_{i}$ com base na Equação 3 .

$$
\begin{gathered}
-l_{1}^{b}+l_{2}^{b}-l_{7}^{b}-v_{1}+v_{2}-v_{7}=0 \\
-l_{6}^{b}+l_{7}^{b}+l_{8}^{b}-v_{6}+v_{7}+v_{8}=0 \\
l_{3}^{b}-l_{4}^{b}-l_{5}^{b}-l_{8}^{b}+v_{3}-v_{4}-v_{5}-v_{8}=0 \\
l_{5}^{b}-l_{9}^{b}+l_{10}{ }^{b}+v_{5}-v_{9}+v_{10}=0 \\
l_{6}^{b}+l_{9}^{b}-l_{11}^{b}+v_{6}+v_{9}-v_{11}=0
\end{gathered}
$$

De acordo com a Equação 8 tem-se:

$$
\begin{gathered}
-v_{1}+v_{2}-v_{7}+w_{1}=0 \\
-v_{6}+v_{7}+v_{8}+w_{2}=0 \\
+v_{3}-v_{4}-v_{5}-v_{8}+w_{3}=0 \\
+v_{5}-v_{9}+v_{10}+w_{4}=0 \\
+v_{6}+v_{9}-v_{11}+w_{5}=0
\end{gathered}
$$

O modelo sendo linear, os coeficientes das incógnitas representam as derivadas parciais, resultando a matriz $B$, Equação 7, de ordem $5 \times 11$.

A partir da Equação 6, obtém-se o vetor erro de fechamento:

$$
W=F\left(L_{b}\right)=\left(\begin{array}{c}
w_{1} \\
w_{2} \\
w_{3} \\
w_{4} \\
w_{5}
\end{array}\right)=\left(\begin{array}{c}
-l_{1}^{b}+l_{2}^{b}-l_{7}^{b} \\
-l_{6}^{b}+l_{7}^{b}+l_{8}^{b} \\
l_{3}^{b}-l_{4}^{b}-l_{5}^{b}-l_{8}^{b} \\
l_{5}^{b}-l_{9}^{b}+l_{10}^{b} \\
l_{6}^{b}+l_{9}^{b}-l_{11}^{b}
\end{array}\right)(m)
$$

A distância de cada seção é representada por $k_{i}$ e o desnível medido por $h_{i}$, dados na Tabela 2. As observações são ditas não correlacionadas e as variâncias são proporcionais às distancias $k_{i} \mathrm{em} \mathrm{km}$. São calculadas as altitudes ajustadas empregando-se a sequência dos passos do MMQ - modelo dos Correlatos, fazendose para isso as seguintes considerações conceituais: primeiro, que as observações 
são independentes, ou seja, a matriz $\mathrm{P}$ é diagonal; segundo: que os pesos sejam inversamente proporcionais aos comprimentos das linhas $\left(P_{i}=1 / k_{i}\right)$.

Definidas as matrizes W e B, obtém-se a matriz dos pesos. Neste trabalho, considera-se a princípio, uma tolerância altimétrica de $\sigma_{i}=0,3 m m \sqrt{k_{i}}$, de acordo com o nível digital empregado, tendo-se assim, $\sigma_{i}^{2}=9 \times 10^{-8} \times k_{i}$; sendo ainda este mesmo valor adotado como o sigma a priori.

Reescrevendo, obtém-se:

$$
P_{n \times n}=\sigma_{0}^{2} \cdot\left(\begin{array}{cccc}
1 / \sigma_{1}^{2} & 0 & \ldots & 0 \\
0 & 1 / \sigma_{2}^{2} & \ldots & 0 \\
0 & 0 & \ldots & 0 \\
0 & 0 & \ldots & 1 / \sigma_{n}^{2}
\end{array}\right)_{n \times n}
$$

Então

$$
\begin{aligned}
P_{11 \times 11} & =k_{i}^{-1}{ }_{11 \times 11} \\
V & =P^{-1} \cdot B^{T} . K
\end{aligned}
$$

na qual $K$ são multiplicadores de Lagrange (correlatos).

Os valores observados ajustados são obtidos a partir do vetor dos resíduos, Equação 20, resultando no vetor $L_{a}$.

Com base nestes experimentos, obteve-se uma variância da observação de peso unitário a posteriori calculada pela Equação 21:

$$
\tilde{\sigma}_{0}^{2}=\frac{V^{T} P V}{S}
$$

Obtendo-se assim, o valor

$$
\widehat{\sigma}_{0}^{2}=5,0741 \times 10^{-6}
$$

Sendo $S$ o número de grau de liberdade.

\subsubsection{Análise da qualidade do ajustamento}

Utilizando-se o teste $\chi^{2}$ Qui-Quadrado apresentado no item 2.2.3, tem-se:

$$
T=\chi_{c}^{2}=\frac{\hat{\sigma}_{0}^{2}}{\sigma_{0}^{2}} S=\frac{5,0741 \times 10^{-6}}{9 \times 10^{-8}} .5=281,8954
$$

com graus de liberdade igual a 5, com os valores teóricos da distribuição $\chi_{(S, 1-\alpha)}^{2}$. A hipótese nula $H_{0}$ não é rejeitada, ao nível de significância $\alpha=0,5 \%$, no teste estatístico, se: $T<\chi_{(S ; 1-\alpha)}^{2}$. Caso contrário, $T>\chi_{(S ; 1-\alpha)}^{2}$, significando que há 
problemas no ajustamento. Portanto, sendo $\chi_{(5 ; 0,995)}^{2}=16,75$, o teste não é aceito, pois: $T=281,8954>\chi_{(5 ; 0,995)}^{2}=16,75$

A hipótese alternativa é rejeitada ao nível de significância de $0,5 \%$.

Utilizando-se o Método de Baarda (cf. item 2.2.3), tem-se: $\sum_{11 \times 11}^{V}=\sigma_{0}^{2} \cdot P_{11 \times 111 \times 5}^{-1} \cdot B^{T} \cdot M_{5 \times 5}^{-1} \cdot B \times{ }_{5 \times 11}^{B} \cdot P_{11 \times 11}^{-1}$

Para a não rejeição de uma hipótese deve-se comparar o valor teórico $\left(-N_{\frac{\alpha_{o}}{2}} ; N_{\frac{\alpha_{o}}{2}}\right)$ com o valor calculado $w$. A estatística $w_{i}$ tem distribuição $\sqrt{F}$ com graus de liberdade $1 \mathrm{e}_{\infty, \sqrt{F_{1, \infty}}}$ ou ${ }_{w_{i}} \sim N_{\frac{\alpha}{2}(0,1)}$

Hipóteses:

$$
\left\{\begin{array}{l}
H_{0}: v_{i}=0 \\
H_{1}: v_{i} \neq 0
\end{array}\right.
$$

A hipótese básica $H_{0}$ não é rejeitada a um determinado nível de significância $\alpha_{o}=\frac{\alpha}{n}=0,00$, se: $-N_{\frac{\omega}{2}}<w_{i}<N_{\frac{\omega o}{2}}$ ou $\quad-\sqrt{\chi_{o, 1}^{2}}<w_{i}<\sqrt{\chi_{o, 1}^{2}}$

Caso contrário, a observação $i$ contém erro grosseiro. Detectado mais de uma observação com erro será eliminada a que representar a maior estatística $\left|w_{i}\right|$, pois o teste é unidimensional. $\mathrm{O}$ teste deve ser repetido até que todas as observações com erro sejam eliminadas. Portanto, o intervalo será:

$-N_{\frac{\omega}{2}}<w_{i}<N_{\frac{\omega}{2}}$, ou seja, $-3,99<w_{i}<3,99$

Com base nos resíduos e suas respectivas incertezas, os resíduos normalizados, descritos na Tabela 3 foram calculados para cada observação empregando-se a Equação 14.

Pode-se observar que 5 observações estão na área de rejeição, conforme Tabela 3, eliminando-se a observação que possui a maior estatística $\left|w_{i}\right|$. A observação que foi eliminada foi a observação $l_{11}$, correspondente à estatística $w_{11}$, desnível de $R N M R$ para $R N 394 D$. Reiniciando-se um novo ajustamento.

Para o novo ajustamento foi retirado o circuito contendo a linha de nivelamento geométrico $l_{l l}$. Neste caso, a variância da unidade de peso a posteriori obteve um valor de: $\widehat{\sigma}_{0}^{2}=3,2221 \times 10^{-7}$. 
O estabelecimento de padrões de referência altimétrica utilizando...

Tabela 3 - Resíduos normalizados.

\begin{tabular}{c|c}
\hline Resíduos Normalizados & $\begin{array}{c}\text { Valores dos resíduos } \\
\text { normalizados }(\mathrm{m})\end{array}$ \\
\hline$w_{1}$ & 3,7720 \\
\hline$w_{2}$ & $-3,7720$ \\
\hline$w_{3}$ & $-0,0126$ \\
\hline$w_{4}$ & 0,0126 \\
\hline$w_{5}$ & 0,0029 \\
\hline$w_{6}$ & $-6,0322$ \\
\hline$w_{7}$ & $-4,5002$ \\
\hline$w_{8}$ & $-8,8451$ \\
\hline$w_{9}$ & $-16,3576$ \\
\hline$w_{10}$ & $-0,0066$ \\
\hline$w_{11}$ & 16,3577 \\
\hline
\end{tabular}

Do mesmo modo como descrito anteriormente é necessário realizar uma comparação entre $\hat{\sigma}_{0}^{2}$ e $\sigma_{0}{ }^{2}$. Neste contexto, o valor calculado para $T$ é: $T=\chi_{c}^{2}=\frac{\hat{\sigma}_{0}^{2}}{\sigma_{0}{ }^{2}} \cdot S=\frac{3,2221 \times 10^{-7}}{9 \times 10^{-8}} \cdot 4=14,3205$, que tem distribuição $\chi^{2}$, com graus de liberdade agora igual a 4 , com os valores teóricos da distribuição $\chi_{(S, 1-\alpha)}^{2}$. A hipótese nula $H_{0}$ não é rejeitada, e o teste é aceito ao nível de significância $\alpha=0,5 \%, T=14,3205<\chi_{(4,0,995)}^{2}=14,86$.

O cálculo da Matriz Variância-Covariância dos resíduos é realizado aplicandose a Equação 15, obtendo-se:

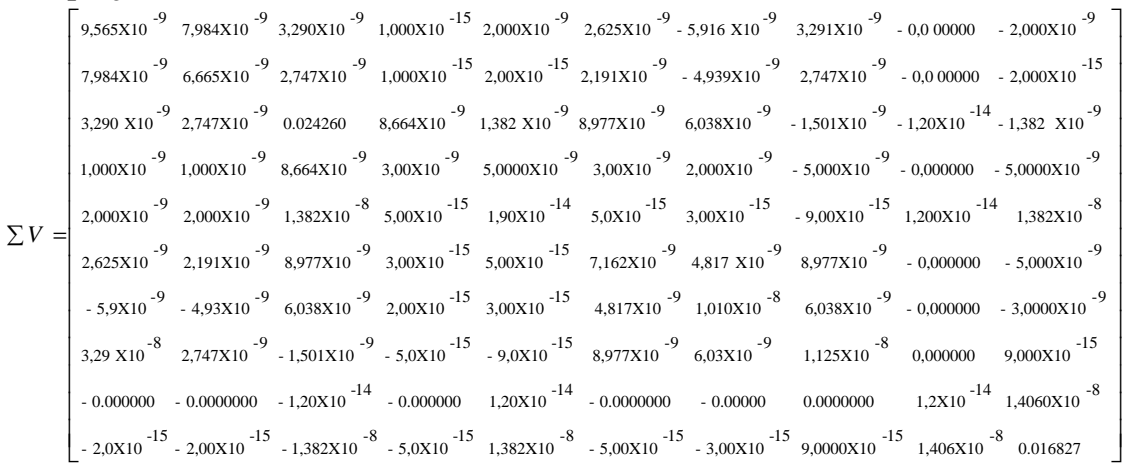

Bol. Ciênc. Geod., sec. Artigos, Curitiba, v. 20, no 2, p.388-410, abr-jun, 2014. 
Os resíduos com suas respectivas incertezas são apresentados na Tabela 4.

Tabela 4 - Resíduos e suas respectivas incertezas.

\begin{tabular}{c|c|c}
\hline Resíduos & $\begin{array}{c}\text { Valores dos } \\
\text { resíduos (m) }\end{array}$ & Incertezas (m) \\
\hline$v_{1}$ & $-0,0002$ & $\pm 9,7802 \times 10^{-5}$ \\
\hline$v_{2}$ & $-0,0001$ & $\pm 8,1644 \times 10^{-5}$ \\
\hline$v_{3}$ & $-0,0013$ & $\pm 0,1557$ \\
\hline$v_{4}$ & 0,0000 & $\pm 5,5629 \times 10^{-8}$ \\
\hline$v_{5}$ & 0,0000 & $\pm 1,3866 \times 10^{-7}$ \\
\hline$v_{6}$ & 0,0003 & $\pm 8,4633 \times 10^{-5}$ \\
\hline$v_{7}$ & $-0,0001$ & $\pm 1,0051 \times 10^{-4}$ \\
\hline$v_{8}$ & $-0,0003$ & $\pm 1,0607 \times 10^{-4}$ \\
\hline$v_{9}$ & 0,0000 & $\pm 1,0839 \times 10^{-7}$ \\
\hline$v_{10}$ & 0,0000 & $\pm 0,1297$ \\
\hline
\end{tabular}

Tomando os resíduos e suas respectivas incertezas, os resíduos normalizados, Tabela 5, foram calculados para cada observação empregando-se a Equação 14.

Tabela 5 - Resíduos normalizados.

\begin{tabular}{c|c}
\hline Resíduos Normalizados & $\begin{array}{c}\text { Valores dos resíduos } \\
\text { normalizados }(\mathrm{m})\end{array}$ \\
\hline$w_{1}$ & 1,9929 \\
\hline$w_{2}$ & $-1,9929$ \\
\hline$w_{3}$ & $-0,0087$ \\
\hline$w_{4}$ & 0,0087 \\
\hline$w_{5}$ & 0,0060 \\
\hline$w_{6}$ & 3,6829 \\
\hline$w_{7}$ & $-1,3693$ \\
\hline$w_{8}$ & $-3,6829$ \\
\hline$w_{9}$ & $-6,1669$ \\
\hline$w_{10}$ & 6,1669 \\
\hline
\end{tabular}

Com base na Tabela 5, observa-se que o menor resíduo normalizado (em módulo) foi igual a $0,0060 \mathrm{~m}$ e o maior (em módulo) foi igual a $6,1669 \mathrm{~m}$. 
3.3.4 Cálculo das altitudes ajustadas

Aplicando-se a propagação de variâncias na expressão $L_{a}=L_{b}+V=L_{b}+P^{-1} B K$ e realizando as operações indicadas, resulta:

$$
\left.\sum L_{a}=\sum L_{b} \mid I-B^{T} M^{-1} B P^{-1}\right\rfloor
$$

com

$$
\sum L_{b}=\sigma_{0}^{2} P^{-1}
$$

Verifica-se que o segundo termo do segundo membro da expressão, Equação 22 , representa a melhoria introduzida com o ajustamento.

O cálculo da Matriz Variância-Covariância das observações ajustadas $\sum L_{a}$ é realizado aplicando-se a Equação 22, obtendo-se:

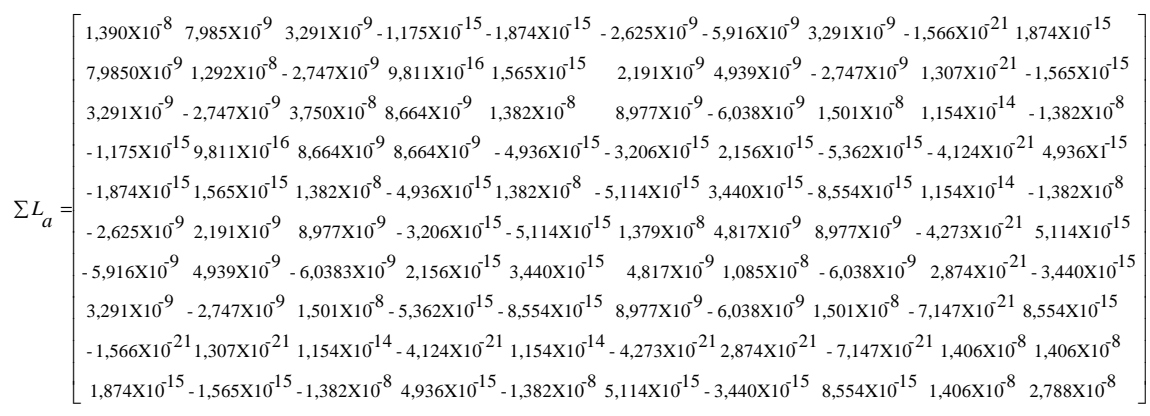

As observações ajustadas são dadas na Tabela 6 .

Tabela 6 - Desníveis ajustados e respectivas incertezas.

\begin{tabular}{c|c|c}
\hline Observções & $\Delta \mathrm{H}^{\mathrm{a}}(\mathrm{m})$ & Incertezas $(\mathrm{m})$ \\
\hline$l_{1}^{a}$ & 7,1858 & $\pm 0,0001$ \\
\hline$l_{2}^{a}$ & 9,8168 & $\pm 0,0001$ \\
\hline$l_{3}^{a}$ & 9,0331 & $\pm 0,0001$ \\
\hline$l_{4}^{a}$ & 7,6610 & $\pm 0,0000$ \\
\hline$l_{5}^{a}$ & 0,8513 & $\pm 0,0001$ \\
\hline$l_{6}^{a}$ & 3,1516 & $\pm 0,0001$ \\
\hline$l_{7}^{a}$ & 2,6309 & $\pm 0,0001$ \\
\hline$l_{8}^{a}$ & 0,5207 & $\pm 0,0001$ \\
\hline$l_{9}^{a}$ & 12,8490 & $\pm 0,0001$ \\
\hline$l_{10}^{a}$ & 11,9977 & $\pm 0,0001$ \\
\hline
\end{tabular}

Bol. Ciênc. Geod., sec. Artigos, Curitiba, v. 20, nº 2, p.388-410, abr-jun, 2014. 
As altitudes ajustadas das RRNN são obtidas, somando-se os respectivos desníveis ajustados, independentemente do "caminho percorrido":

$$
\begin{gathered}
H_{2}=H_{1}-l_{1}^{a} \\
H_{3}=H_{1}-l_{1}^{a}+l_{2}^{a} \\
H_{4}=H_{1}-l_{1}^{a}+l_{2}^{a}+l_{3}^{a} \\
H_{5}=H_{1}+l_{6}^{a}+l_{5}^{a} \\
H_{6}=H_{1}+l_{6}^{a} \\
H_{7}=H_{1}+l_{6}^{a}+l_{9}^{a}
\end{gathered}
$$

A Matriz Variância - Covariância das altitudes corrigidas $\sum H$ é obtida através da propagação nas expressões anteriores, ou seja,

$$
\sum H=G \cdot \sum L_{a} \cdot G^{T}
$$

Sendo a matriz $\mathrm{G}$ dos coeficientes:

$$
G_{6 \times 10}=\left(\begin{array}{cccccccccc}
-1 & 0 & 0 & 0 & 0 & 0 & 0 & 0 & 0 & 0 \\
-1 & 1 & 0 & 0 & 0 & 0 & 0 & 0 & 0 & 0 \\
-1 & 1 & 1 & 0 & 0 & 0 & 0 & 0 & 0 & 0 \\
0 & 0 & 0 & 0 & 1 & 1 & 0 & 0 & 0 & 0 \\
0 & 0 & 0 & 0 & 0 & 1 & 0 & 0 & 0 & 0 \\
0 & 0 & 0 & 0 & 0 & 1 & 0 & 0 & 1 & 0
\end{array}\right)_{6 \times 10}
$$

Substituindo a MVC das observações ajustadas $\sum L_{a}$ e a matriz G dos coeficientes, resulta que: $\sum_{6 \times 6} H=\underset{6 \times 10}{G} \cdot \sum_{10 \times 10} L_{a} \cdot G_{10 \times 6}^{T}\left(m^{2}\right)$

A Tabela 7 apresenta as altitudes ajustadas, calculadas pelas equações descritas acima (em metros):

Tabela 7 - Altitudes ajustadas e suas incertezas com nível de confiança de 99,5\%.

\begin{tabular}{c|c|c}
\hline $\begin{array}{c}\text { Referência de } \\
\text { Nível }\end{array}$ & $\begin{array}{c}\text { Altitudes } \\
\text { ajustadas }(\mathrm{m})\end{array}$ & Incertezas (m) \\
\hline$R N S C\left(H_{2}\right)$ & 8,7224 & $\pm 0,0001$ \\
\hline$R N L S E\left(H_{3}\right)$ & 18,5392 & $\pm 0,0001$ \\
\hline$R N L M\left(H_{4}\right)$ & 27,5723 & $\pm 0,0001$ \\
\hline$R N 7 E\left(H_{5}\right)$ & 19,9111 & $\pm 0,0001$ \\
\hline$R N P M\left(H_{6}\right)$ & 19,0598 & $\pm 0,0001$ \\
\hline$R N M R\left(H_{7}\right)$ & 31,9088 & $\pm 0,0001$ \\
\hline
\end{tabular}




\section{CONCLUSÕES}

As estruturas geodésicas altimétricas, implantadas e determinadas pelo método do nivelamento geométrico, materializam, neste trabalho, pontos que servem de padrões de referência altimétrica para a determinação altimétrica de alvos altos e inacessíveis, localizados nas Torres das Igrejas observadas por meio do método do nivelamento trigonométrico. Este trabalho tem como objetivo definir padrões de referência altimétrica utilizando o método de nivelamento geométrico, resultando na implantação e análise da qualidade de estruturas geodésicas altimétricas no Sítio Histórico de Olinda.

O ajustamento foi realizado pelo método dos Mínimos Quadrados, utilizando o modelo dos Correlatos. A técnica denominada Data Snooping, que utiliza a convenção de que somente um outlier está presente em cada teste envolvendo várias observações detectou um erro grosseiro na linha de nivelamento $l_{11}$, realizada da $R N M R$ para a $R N 394 D$. Uma vez eliminados os erros grosseiros ou outliers, refez-se o ajustamento, o qual foi aceito pelo Teste Global do Modelo. A hipótese básica $H_{0}$ não foi rejeitada ao nível de significância de $\alpha=0,5 \%$; o valor calculado para a forma quadrática $\mathrm{V}^{\mathrm{T}} \mathrm{PV}$ tem distribuição Qui-Quadrado calculado com 4 graus de liberdade, de 14,3205 e está entre os valores tabelados de 0,21 e 14,86. O maior resíduo encontrado refere-se à observação $l_{3}$ com valor de $-0,0013 \mathrm{~m}$ e menor para a observação $l_{4}$ com valor igual a zero; uma vez que os resíduos são proporcionais aos pesos, estes não possuem distribuição normal, porém, com a padronização, estes apresentaram uma distribuição normal, o que indica que não há problemas quanto ao ajustamento.

As estruturas geodésicas implantadas no Sítio Histórico de Olinda auxiliarão nos trabalhos que envolvem estudos de deslocamentos verticais, reconstrução tridimensional de objetos, levantamentos topográficos/geodésicos, levantamentos cadastrais, monitoramento de edificações prediais, assim como a definição de um modelo geoidal local acurado. Estas estruturas geodésicas beneficiarão estudos desenvolvidos por algumas instituições como: UFPE, Prefeitura Municipal de Olinda, IPHAN e Defesa Civil.

Cabe salientar que o não conhecimento da precisão da RN394D compromete a confiabilidade das altitudes dos pontos de referência altimétrica determinados com relação ao Sistema de Referência Vertical Brasileiro. Portanto, recomenda-se a vinculação da RN394D a RRNN com precisões conhecidas.

A metodologia apresentada neste trabalho está sendo aplicada atualmente no Centro Histórico de João Pessoa (PB) no projeto denominado "A Contribuição da Geodésia Aplicada na Discretização de Edificações Prediais" do edital CNPq/VALE S.A. Forma - Engenharia 05/2012 em andamento, envolvendo a Universidade Federal de Pernambuco (UFPE) em parceria com o Instituto Federal de Educação, Ciência e Tecnologia da Paraíba (IFPB), com o objetivo da representação 3D das edificações históricas. 


\section{AGRADECIMENTOS}

Ao Departamento de Engenharia Cartográfica pela concessão dos equipamentos e Laboratórios LAGEO e LATOP e ao Curso de Pós-Graduação pela concessão de recursos para a realização do trabalho. Ao Projeto CNPq/VALE S.A Forma - Engenharia nº 05/2012. Ao Projeto DLR/FADE/UFPE 2009.

\section{REFERÊNCIAS BIBLIOGRÁFICAS}

ABNT. NBR-13.133 - Normas Técnicas para a Execução de Levantamentos Topográficos. Rio de Janeiro, 1994.

BAARDA, W. A testing procedure for use in geodetic networks. Netherlands Geodetic Commission. Publications on Geodesy. Série 2. N. 5. Delft, Netherlands, $1968,97 \mathrm{p}$.

BOTELHO, F. J. L. Métodos de Racionalização Construtiva no Posicionamento preciso de Edificações Prediais. Dissertação apresentada ao Programa de PósGraduação em Ciências Geodésicas e Tecnologia da Geoinformação, 2003.

DEUMILICH. F.; STAIGER, R. Instrumentenkunde der Vermessungstechnik. Aufl. 9. Wichmann, 2002.

DREWES, H.; SÁNCHEZ, L.; BLITZKOW, D.; FREITAS, S.R.P. Documento técnico sobre o sistema de referência vertical para a América do Sul. SIRGAS, Boletim Informativo n. 6, 1998, p. 23-31.

FRASER, C. S. Least-Squares \& Network Analysis. Class Notes. University of Melbourne. Australia, 2003.

FREITAS, S.R.C.; BLITZKOW, D. Altitudes e geopotencial. IGES Bulletin Special Issue for South America, n. 9, 1999, p. 47-62.

GAMA, L. F. Experimentos e Análises Metodológicas do Desempenho de Estruturas Geodésicas Planimétricas Implantadas com GPS e Estação Total: Aplicações em Levantamentos Cadastrais Urbanos. Dissertação de Mestrado apresentado ao Programa de Pós-Graduação em Ciências Geodésicas e Tecnologias da Geoinformação da UFPE. Recife. 2008.

GEMAEL, C. Introdução ao Ajustamento de Observações: aplicações geodésicas. Curitiba: Ed.UFPR,1994. Reimpressão 2004.

GUIMARÃES, G. N. A geoid model in state of São Paulo: an attempt for the evaluation of diferente methodologies. Tese de Doutorado - Escola Politécnica da Universidade de São Paulo. Departamento de Engenharia de Transportes. São Paulo, 2013.

IBGE. Resolução - PR $n^{o}$ 22, de 21-07-83 - Especificações e Normas Gerais para Levantamentos Geodésicos, 1983 - www.ibge.gov.br (acessado em 02/07/2011).

JORDAN, D. W. Tratado general de Topografia. V. I. Barcelona, Editorial Gustavo Gili, S. A.,1944.

KAHMEN, H. Angewandete Geodaesie. Vermessungskunde.20. Aufl., 2005.

KRARUP, T.; KUBIK, K.; The Danish Method for adjustment; discussion. In: Suplement to the Proceedings - Symposium Mathematical Models, Accuracy 
Aspects and Quality Control, Otaniemi, Helsinki University of Tecnology, p.26-29, 1982.

LEICA. Manual do utilizador Leica DNA 03/DNA 10. Versão 2.0 - português, 2002.

MATOS, A. C. O. C.; BLITZKOW, D.; GUIMARÃES, G. N.; LOBIANCO, M. C.

B.; COSTA, S. M. C. Validação do MAPGEO2010 e Comparação com Modelos do Geopotencial Recentes. Bol. Ciênc. Geod., sec. Artigos, Curitiba, v. 18, n 1 , p.101-122, jan-mar, 2012.

MENDONÇA, F. J. B.; GARNÉS, S. J. dos A.; PEREIRA, C. M.; NETO, J. A. B.; MELO, W. D. A. Análise do ajustamento por mínimos quadrados de uma trilateração topográfica com injunções nos planos UTM e Topocêntrico. III Simpósio Brasileiro de Ciências Geodésicas e Tecnologias da Geoinformação, Recife-PE, 2010.

MIKHAIL, E. M.; GRACIE, G. Analysis and adjustment of survey measurements. New York: Van Nostrand Reinhold Company, 1981.

MONICO, J. F. G. Posicionamento pelo GNSS: descrição, fundamentos e aplicações. 2 ed. - São Paulo: Editora UNESP, 2008.

MONICO, J.F.G.; SILVA, E.F. Controle de Qualidade em Levantamentos no Contexto da Lei $\mathrm{n}^{\circ} 10.267$ de 28 de agosto de 2001. In: Série em Ciências Geodésicas. Vol.3 Curitiba:Ed. Edson Aparecido Mitishita.2003.

NIEMEIER, W. Ausgleichungsrechnung. New York: De Gruyter Lehrbuch.2002.

POPE, A. J. The statistics of residuals and the detection of outliers. NOAA Technical Rep. NOS 65 NGS 1, U.S. National Geodetic Survey, Silver Spring, Md, 1976, 136p.

RHADAMEK, A. A. P. Uma Abordagem Metodológica para a Identificação, Representação e Monitoramento Geodésico de uma Encosta em Risco Natural. Dissertação de Mestrado apresentado ao Programa de Pós-Graduação em Ciências Geodésicas e Tecnologias da Geoinformação da UFPE. Recife, 2008.

SANTOS, S. M. dos. Investigações Metodológicas sobre o monitoramento da subsidência do solo devido à extração de água subterrânea - Caso da Região Metropolitana de Recife. Tese de Doutorado apresentada ao Programa de PósGraduação em Engenharia Civil UFPE. Recife, 2005.

SOUZA, A. M. B. Análise e comparação de estruturas geodésicas tridimensionais definidas por métodos planialtimétricos de medição. Dissertação de mestrado apresentado ao Programa de Pós-Graduação em Ciências Geodésicas e Tecnologias da Geoinformação da UFPE. Recife. 2012.

SEIXAS, A. De.; SOUZA, A. M. B.; GAMA,L.F. Implementation and Determination of the Three-Dimensional Geodesic Structures in the Olinda's Historical Site. FIG Working Week 2013 Environment for Sustainability Abuja, Nigeria. 2013.

TORGE, W. Geodaesie, 2. Aufage, Berlin: de Gruyter Lehrbuch, 2003.

( Recebido em setembro de 2013. Aceito em fevereiro de 2014).

Bol. Ciênc. Geod., sec. Artigos, Curitiba, v. 20, no 2, p.388-410, abr-jun, 2014. 